UUITP-06/12

AEI-2012-019

\title{
New Relations for Three-Dimensional Supersymmetric Scattering Amplitudes
}

\author{
Till Bargheer,${ }^{a}$ Song He, ${ }^{b}$ And Tristan McLoughlin ${ }^{b}$ \\ ${ }^{a}$ Department of Physics and Astronomy, Uppsala University, SE-751 08 Uppsala, Sweden \\ ${ }^{b}$ Max-Planck-Institut für Gravitationsphysik, Albert-Einstein-Institut, Am Mühlenberg 1, \\ D-14476 Potsdam, Germany
}

till.bargheer@physics.uu.se, \{song.he,tmclough\}@aei.mpg.de

\begin{abstract}
We provide evidence for a duality between color and kinematics in threedimensional supersymmetric Chern-Simons matter theories. We show that the six-point amplitude in the maximally supersymmetric, $\mathcal{N}=8$, theory can be arranged so that the kinematic factors satisfy the fundamental identity of three-algebras. We further show that the four- and six-point $\mathcal{N}=8$ amplitudes can be "squared" into the amplitudes of $\mathcal{N}=16$ three-dimensional supergravity, thus providing evidence for a hidden three-algebra structure in the dynamics of the supergravity.
\end{abstract}

\section{Introduction}

Scattering amplitudes have provided a rich vein of insight into the hidden structures underlying our theories of gauge and gravitational interactions. One particularly suggestive result is the color-kinematics duality discovered by Bern, Carrasco, and Johansson (BCJ) [1]. At treelevel, color dressed scattering amplitudes in Yang-Mills (YM) theories can, quite generally, be written as a sum over cubic graphs

$$
\mathcal{A}_{n}=g^{n-2} \sum_{i \in \text { graphs }} \frac{n_{i} c_{i}}{\prod_{\ell_{i}} p_{\ell_{i}}^{2}},
$$

where the $c_{i}$ 's are color structures made from the usual Lie algebra structure constants, and the $n_{i}$ 's are kinematic factors from which we have removed products of inverse propagators $p_{\ell_{i}}^{2}$ associated to internal lines of the respective cubic diagram. BCJ proposed that there exists a representation of the amplitude such that for any set of color structures related by a Jacobi identity, there is a corresponding relation between their numerator factors, i.e.

$$
c_{1}+c_{2}+c_{3}=0 \Rightarrow n_{1}+n_{2}+n_{3}=0 .
$$

This duality implies non-trivial relations between different tree-level color-ordered subamplitudes, so-called BCJ relations [1].

Moreover, as is well known, via the Kawai-Lewellen-Tye (KLT) relations [2, 3], such Yang-Mills amplitudes can be used to express tree-level scattering in related gravity theories. 
BCJ [1, 4] proposed that it is possible to express gravity amplitudes in terms of the gauge theory data by simply replacing the color factors by another copy of the kinematic numerators and summing over the same cubic diagrams.

In this note, we provide evidence for a non-trivial analogue of the color-kinematics duality in supersymmetric Chern-Simons matter (SCS) theories and for a corresponding "doublecopy" construction leading to the $E_{8(8)}$ symmetric, three-dimensional $\mathcal{N}=16$ supergravity.

\section{$2 \mathcal{N}=8$ SCS scattering amplitudes}

The maximally supersymmetric Chern-Simons theory, the Bagger-Lambert-Gustavsson (BLG) theory, constructed in [5, 6, 7], is the unique three-dimensional gauge theory with $O S p(8 \mid 4)$ superconformal symmetry. The on-shell, physical states comprise eight scalars, $X^{I}$, in the $\mathbf{8}_{\mathbf{v}}$ of $S O(8)$ and eight fermions, $\Psi^{\dot{I}}$, in the $\mathbf{8}_{\mathbf{c}}$. An important feature of the original construction was the appearance of three-algebras. Briefly, a three-algebra is a vector space, $T^{a}, a=1, \ldots, N$, with a trilinear product

$$
\left[T^{a}, T^{b}, T^{c}\right]=f_{d}^{a b c} T^{d},
$$

where the structure constants $f_{d}^{a b c}$ satisfy the fundamental three-algebra identity,

$$
f_{d}^{e f g} f_{g}^{a b c}=f_{g}^{e f a} f_{d}^{b c g}+f_{g}^{e f b} f_{d}^{c a g}+f_{g}^{e f c} f_{d}^{a b g} .
$$

Moreover there is a trace form, $h^{a b}=\operatorname{Tr}\left(T^{a} T^{b}\right)$, which can be used to raise and lower indices. The structure constants with all indices raised are completely anti-symmetric, $f^{a b c d}=f^{[a b c d]}$. All on-shell fields are three-algebra valued fundamental fields, e.g. $X^{I}=\sum_{a=1}^{N}\left(X^{I}\right)^{a} T^{a}$. The only known finite-dimensional example is where the three-algebra is four-dimensional, while the structure constants are proportional to the invariant four-index tensor $f^{a_{1} a_{2} a_{3} a_{4}} \propto$ $\epsilon^{a_{1} a_{2} a_{3} a_{4}}$.

As we are interested in scattering amplitudes, it is convenient to make use of the spinorhelicity formalism, whereby three-momenta are expressed as the product of two-component real spinors: ${ }^{1} p^{\alpha \beta}=\lambda^{\alpha} \lambda^{\beta}$ where $\alpha, \beta=1,2$. The on-shell fields can be grouped into a single superfield ${ }^{2}$ by introducing four Graßmann parameters $\gamma^{i}, i=1, \ldots, 4$. This construction breaks manifest $S O(8)$ R-symmetry by rewriting the $\mathbf{8}_{\mathbf{v}}$ scalars as $X^{I}=\left\{\bar{X}, X^{[i j]}, X\right\}$ and similarly for the fermions, $\Psi^{\dot{A}}=\left\{\psi_{i}, \bar{\psi}^{i}\right\}$, so that the on-shell superfield is

$$
\begin{aligned}
\Phi_{\mathrm{BLG}}= & \bar{X}+\gamma^{i} \psi_{i}+\frac{1}{2} \epsilon_{i j k l} \gamma^{i} \gamma^{j} X^{[k l]} \\
& +\frac{1}{3 !} \epsilon_{i j k l} \gamma^{i} \gamma^{j} \gamma^{k} \bar{\psi}^{l}+\frac{1}{4 !} \epsilon_{i j k l} \gamma^{i} \gamma^{j} \gamma^{k} \gamma^{l} X .
\end{aligned}
$$

$O S p(8 \mid 4)$ invariant four-point scattering amplitudes,

$$
\mathcal{A}_{4}=\frac{4 \pi i}{k} \frac{\delta^{(3)}(P) \delta^{(8)}(Q)}{\langle 12\rangle\langle 23\rangle\langle 31\rangle} f^{a_{1} a_{2} a_{3} a_{4}},
$$

have previously been constructed [9]. In this formula, the delta-functions impose conservation of momenta, $P^{\alpha \beta}=\sum_{j=1}^{4} p_{j}^{\alpha \beta}$, and supermomenta, $Q^{\alpha i}=\sum_{j=1}^{4} \lambda_{j}^{\alpha} \gamma_{j}^{i}$, while the kinematic

\footnotetext{
${ }^{1}$ In fact, only particles with positive energy correspond to real spinors. For negative energies, $\lambda$ is taken to be purely imaginary.

${ }^{2}$ Our construction closely parallels the oscillator construction of the $O S p(8 \mid 4)$ algebra 8 , and so is only $U(4 \mid 2)$ covariant, corresponding to the Jordan decomposition of $O S p(8 \mid 4)$ with respect to a $U(1) \in U(4 \mid 2)$. An equivalent on-shell superfield formulation of BLG was constructed in 9 .
} 


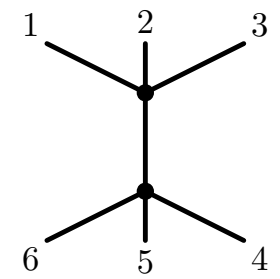

Figure 1: Six point quartic diagram.

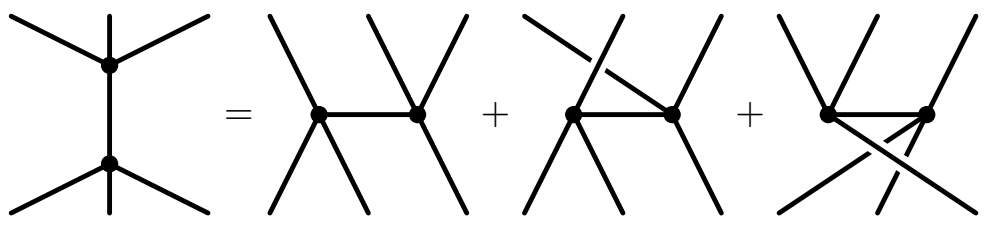

Figure 2: Graphical expression of the fundamental identity.

invariants are defined as $\langle j k\rangle=\epsilon_{\alpha \beta} \lambda_{j}^{\alpha} \lambda_{k}^{\beta}$. The overall form of the amplitude is fixed by the superconformal symmetries, while the normalization, dependence on the Chern-Simons coupling $k$, and color structure, are fixed by explicit Feynman diagram calculation of any component amplitude.

Quite generally we can write an $n$-point amplitude in the BLG theory in the form (1), but with the $c_{i}$ corresponding to three-algebra color structures ${ }^{3}$ The sum is now over diagrams with quartic vertices, and the color structures are found by associating to each vertex a factor $f^{a b c d}$, and to each internal line a metric $h_{a b}$. For example, Fig. 1 corresponds to $c_{(123)(456)}:=f^{a_{1} a_{2} a_{3} b} h_{b c} f^{c a_{4} a_{5} a_{6}}$.

A key feature is that due to the fundamental identities (3) not all of the color structures are independent. Namely, given $c_{s}=\ldots f^{e f g} f_{d}^{a b c} \ldots, c_{t}=\ldots f^{e f a}{ }_{g} f^{b c g}{ }_{d} \ldots, c_{u}=$ $\ldots f^{e f b} f_{g}{ }^{c a g}{ }_{d} \ldots$ and $c_{v}=\ldots f^{e f c} f_{g}^{a b g}{ }_{d} \ldots$, where the "..." denote factors common to all diagrams, then $c_{s}=c_{t}+c_{u}+c_{v}$. Our first proposal is that corresponding numerators $n_{s}, n_{t}$, $n_{u}$ and $n_{v}$ can always be found such that (see Fig. 2)

$$
c_{s}=c_{t}+c_{u}+c_{v} \Rightarrow n_{s}=n_{t}+n_{u}+n_{v} .
$$

We do not have a general proof for these relations, instead we will provide evidence for their existence by considering the first non-trivial case, i.e. six points.

At six points, all color structures consist of the contractions of two tensors as in Fig. 1. Accounting for the anti-symmetry of $f^{a b c d}$, there are ten distinct color structures $c_{i}$, labeled by partitions of the six color labels into groups of three, e.g. $c_{1}=c_{(123)(456)} \cdot{ }^{4}$ At six points there are five independent three-algebra relations between different color structures. Our claim is that there is a choice of numerators such that they satisfy the same three-algebra fundamental identities, however the numerators are not uniquely defined and finding explicit forms is not straightforward. Instead, we will show the existence of such numerators, and give a recipe for calculating them, by considering the color-ordered subamplitudes of $\mathcal{N}=6$ Aharony-Bergman-Jafferis-Maldacena (ABJM) theory [10].

\footnotetext{
${ }^{3}$ Note that the gauge field is non-dynamical and thus only fundamental matter fields appear as external states.

${ }^{4}$ To be explicit we will also choose $c_{2}=c_{(156)(234)}, c_{3}=c_{(612)(345)}, c_{4}=c_{(125)(436)}, c_{5}=c_{(136)(245)}$, $c_{6}=c_{(145)(236)}, c_{7}=c_{(124)(356)}, c_{8}=c_{(143)(256)}, c_{9}=c_{(146)(235)}, c_{10}=c_{(135)(246)}$. We use the same notation for labeling the numerators.
} 


\section{New relations for color-ordered subamplitudes}

As is well known, the BLG theory can be rewritten [11] as a special case $(N=2)$ of the $S U(N) \times S U(N) \mathcal{N}=6$ Chern-Simons theories with bi-fundamental matter, that is ABJMtheories. The ABJM on-shell fields can be grouped into two superfields, $\hat{\Phi}_{\bar{A}}^{A}$, transforming as $(N, \bar{N})$, and $\hat{\bar{\Phi}}_{B}^{\bar{B}}$, transforming as $(\bar{N}, N)$ [12. This formalism is manifestly $U(3)$ symmetric, making use of three Graßmann parameters $\gamma^{\hat{i}}, \hat{i}=1,2,3$. For $N=2$ the conjugate representations are equivalent and the two superfields can be combined: $\Phi_{\mathrm{BLG}}=\hat{\Phi}+\gamma^{4} \hat{\bar{\Phi}}$.

Scattering amplitudes in BLG theory can be found from those of ABJM by identifying the appropriate fields and color structures. ABJM scattering amplitudes can however be decomposed into color-ordered subamplitudes. Each color-ordered subamplitude will contribute to several kinematical coefficients of the BLG color structures $c_{i}$. We claim that every color-ordered ABJM subamplitude can be written as a certain combination of numerators $n_{i}$ with propagators, in such a way that the corresponding BLG amplitudes take the form (1), with the numerators satisfying the three-algebra identities (6). This implies non-trivial relations among the color-ordered ABJM subamplitudes, and thus is a slightly stronger claim than the proposition that the BLG amplitudes decompose as (1) with (6) satisfied. In the following, we provide evidence for this proposal by examining the six-point amplitudes.

Four-point amplitudes in $\mathrm{ABJM}^{5}$ were considered in [13], the six-point color-ordered subamplitude for ABJM were first calculated in [12], see also [14]. As a representative component amplitude, we consider the six-point amplitude involving a single flavor of complex scalar $\phi(p)_{\bar{A}}^{A}$ and its conjugate $\bar{\phi}(p)_{B}^{\bar{B}}$,

$$
\hat{A}_{6 \phi}=A(1,2,3,4,5,6) \delta_{\bar{A}_{1}}^{\bar{B}_{2}} \delta_{B_{2}}^{A_{2}} \delta_{\bar{A}_{3}}^{\bar{B}_{4}} \delta_{B_{4}}^{A_{5}} \delta_{\bar{A}_{5}}^{\bar{B}_{6}} \delta_{B_{6}}^{A_{1}}+\ldots
$$

with the ellipses denoting other color orderings.

At six points, we propose that the color-ordered ABJM subamplitudes take the form

$$
A(i, j, k, p, q, r)=\frac{n_{(i j k)(p q r)}}{p_{i j k}^{2}}+\frac{n_{(q r i)(j k p)}}{p_{q r i}^{2}}+\frac{n_{(r i j)(k p q)}}{p_{k p q}^{2}} .
$$

There are six independent subamplitudes, all others are related to those by cyclic doubleshifts and by inversions, e.g.

$$
\begin{aligned}
& A(3,4,5,6,1,2)=A(1,2,3,4,5,6), \\
& A(1,2,5,6,3,4)=A(1,4,3,6,5,2) .
\end{aligned}
$$

If, as we claim, it is possible to choose numerators satisfying (6), we can solve for five of the numerators, for example by setting

$$
\begin{gathered}
n_{9}=-n_{1}+n_{10}+n_{4}, \quad n_{8}=-n_{2}+n_{3}+n_{4} \\
n_{7}=n_{1}-n_{3}-n_{4}, \quad n_{6}=n_{10}-n_{2}+n_{4} \\
n_{5}=-n_{1}+n_{10}+n_{3}+n_{4} .
\end{gathered}
$$

We can now solve for four further numerators $n_{2}, n_{3}, n_{4}$ and $n_{10}$ in terms of known expressions [12, 14] for $A(1,2,3,4,5,6), A(1,2,3,6,5,4), A(1,2,5,4,3,6)$, and $A(1,4,3,6,5,2)$. We thus derive identities for $A(1,4,5,2,3,6)$ and $A(1,4,3,2,5,6)$ in terms of these subamplitudes and the undetermined numerator $n_{1}$. The expressions for the numerators and correspondingly the identities are rather complicated, however it is straightforward to numerically check, by choosing explicit numerical values for external momenta, that they are

\footnotetext{
${ }^{5}$ Actually of a one parameter family of mass deformed theories.
} 
in fact satisfied. Importantly, the undetermined kinematical factor, $n_{1}$, does not appear in any of these relations and so corresponds to a generalized gauge freedom analogous to that found in the YM case [1].

\section{$4 \quad E_{8(8)}$ supergravity theory}

The three-dimensional $\mathcal{N}=16$ supergravity with $E_{8(8)}$ symmetry ( $E_{8}$-theory), originally constructed by Marcus and Schwarz [15], consists of 128 scalar bosons and 128 fermions which are in inequivalent real spinor representations of $S O(16)$, the maximal compact subgroup of $E_{8(8)}$. An immediate consequence of this, as explained in [15], is that non-trivial scattering amplitudes must have an even number of external particles, as products of odd numbers of spinors cannot form a singlet. Consequently the S-matrix is naively different than the dimensional reduction of the four-dimensional $\mathcal{N}=8$ supergravity with $E_{7(7)}$ symmetry ( $E_{7}$-theory). However, as is long known, e.g. [16], on-shell the two theories are related by performing a duality transformation, after dimensional reduction, of all the vector fields into scalars, which then combine with the scalars from dimensional reduction, including those originally in the $E_{7(7)} / S U(8)$ coset of the $\mathcal{N}=8$ supergravity, to become those of the $E_{8(8)} / S O(16)$ coset.

The $E_{8(8)}$ algebra comprises 120 compact $S O(16)$ generators $X^{I J}, I, J=1, \ldots, 16$, and 128 non-compact generators $Y^{A}, A=1, \ldots, 128$.

It is convenient to fix the unitary-gauge, whereby a generic group element is written as $g=e^{\varphi^{A} Y^{A}}$ with $\varphi^{A}$ the physical scalars. The $E_{8(8)} / S O(16)$-coset action is constructed from the algebra-valued current $P_{\mu}=\frac{1}{2}\left(e^{-\varphi} \partial e^{\varphi}-e^{\varphi} \partial e^{-\varphi}\right)$. The bosonic action is [15],

$$
\mathcal{L}_{\text {bos }}=\frac{1}{4 \kappa^{2}} \sqrt{-g} R-\frac{1}{4 \kappa^{2}} \sqrt{-g} g^{\mu \nu} P_{\mu}^{A} P_{\nu}^{A},
$$

where the first term is the usual gravity action. Using this action (the fermionic terms are also known), with appropriate gauge fixing, one can straightforwardly calculate scattering amplitudes using Feynman diagrams. At four-points such amplitudes for four scalars receive contributions from graviton exchange and from contact interactions that arise upon expanding the coset term to quartic order in fields, $\mathcal{L}_{\varphi^{4}} \sim\left(\varphi \Gamma^{I J} \partial_{\mu} \varphi\right)\left(\varphi \Gamma^{I J} \partial_{\mu} \varphi\right)$. In the simplest case we can consider the scattering of four scalars all carrying the same coset index, e.g. all fields being $\varphi^{1}$, in which case there is no contribution from contact terms. Combining all graviton exchange diagrams we find

$$
M_{4}=\frac{i \kappa^{2}}{4}\left(\frac{s^{2}+u^{2}}{t}+\frac{t^{2}+u^{2}}{s}+\frac{s^{2}+t^{2}}{u}\right) .
$$

It is not difficult to calculate other component amplitudes, however we can make use of the supersymmetry to determine the full four-point superamplitude.

For the $E_{8}$-theory we can define an on-shell superfield by using eight Graßmann parameters $\eta^{I}, I=1, \ldots, 8$ which breaks the $S O(16)$ R-symmetry to $U(8)$. Splitting the 128 scalars $\varphi^{A}$ into the fields $\left\{\xi, \bar{\xi}, \xi_{I J}, \bar{\xi}^{I J}, \xi_{I J K L}\right\}$ with, for example $\xi=\frac{1}{2}\left(\varphi^{1}+i \varphi^{2}\right)$, and similarly for the fermionic fields, we can write the superfield ${ }^{6}$

$$
\Xi=\xi+\eta^{I} \psi_{I}+\frac{1}{2} \eta^{I} \eta^{J} \xi_{I J}+\cdots+\frac{1}{8 !} \eta^{8} \bar{\xi} .
$$

\footnotetext{
${ }^{6}$ This superfield is very similar to that of the $E_{7}$-theory and indeed making the formal identification $\xi=h$, $\bar{\xi}=\bar{h}, \xi_{I J}=B_{I J}, \bar{\xi}^{I J}=\bar{B}^{I J}, \xi_{I J K L}=D_{I J K L}$ to the fields of the $E_{7}$-theory this becomes more apparent.
} 
By using super-Poincaré symmetry and matching to the component amplitude, the fourpoint superamplitude is

$$
\mathcal{M}_{4}=\frac{i \kappa^{2}}{4} \frac{\delta^{(16)}(Q) \delta^{(3)}(P)}{(\langle 12\rangle\langle 23\rangle\langle 31\rangle)^{2}}
$$

Here, the 16-dimensional fermionic delta-function is given by the product of two eightdimensional fermionic delta-functions, $\delta^{(16)}(Q) \sim \delta^{(8)}\left(Q^{1}\right) \delta^{(8)}\left(Q^{2}\right)$, such as appeared in (5). Stripping off the overall normalization and momentum delta-function we see that this is the "square" of (5). This then suggests an analogue of the KLT relation [2, 3] between $\mathcal{N}=4$ supersymmetric Yang-Mills (SYM) and the $E_{7}$ supergravity theory to one between $\mathcal{N}=8$ BLG and the $E_{8}$-theory. As zeroth order checks, we note that the spectra of the $E_{8}$-theory and that of the BLG theory squared match; furthermore in both cases all non-trivial amplitudes have even numbers of legs. Of course the direct dimensional reduction of $\mathcal{N}=4 \mathrm{SYM}$ and the $E_{7}$-theory amplitudes to three dimensions are related by the usual KLT relations, and for fields which are unchanged by the duality transformation, in particular the scalars originating in the $E_{7(7)} / S U(8)$ coset, the three-dimensional scattering amplitudes are just those of the four-dimensional theory evaluated on three-dimensional kinematics. However, after the duality transformation to the $E_{8}$-theory, this ceases to be the case for all amplitudes; as a simple example there is no $E_{8}$-theory three-point amplitude corresponding to the square of the three-dimensional SYM three-point amplitude. As $\mathcal{N}=8$ BLG theory can be found from supersymmetric three-dimensional Yang-Mills [17] via a "Higgsing"-procedure reminiscent of the duality transformation, it is perhaps not surprising that it should be thus related to the $E_{8}$ supergravity theory.

\section{Three-dimensional gravity as the square of Chern-Simons}

Given the suggestion that the BLG amplitudes can be written in terms of numerators satisfying the three-algebra color-kinematics duality, it is natural to ask if the gravity theory amplitudes can be written as a "double-copy" as in [1],

$$
M_{n}=i\left(\frac{\kappa}{2}\right)^{n-2} \sum_{i} \frac{n_{i} n_{i}}{\prod_{\ell_{i}} p_{\ell_{i}}^{2}}
$$

where the $n_{i}$ 's are the numerators appearing in the BLG amplitude (1) and the sum is over the same $n$-point quartic diagrams. This relation obviously holds at four points for the superamplitudes, and at six points we can perform an explicit check by making use of the numerators calculated from the six-point color-ordered ABJM subamplitudes for specific components. For example, the pure scalar ABJM amplitude $\hat{A}_{6 \phi}(7)$ can be used to calculate the numerators for the $A_{6}\left(X_{1} \bar{X}_{2} X_{3} \bar{X}_{4} X_{5} \bar{X}_{6}\right)$ in the BLG theory, which potentially squares into the $M_{6}\left(\xi_{1} \bar{\xi}_{2} \xi_{3} \bar{\xi}_{4} \xi_{5} \bar{\xi}_{6}\right)$ gravity amplitude. That this is indeed the case can in principle be shown by comparing with the result of a direct Feynman diagram calculation. Equivalently, but significantly more efficiently, one can take this complex scalar to have originated in the $E_{7(7)} / S U(8)$ coset, so that the squared amplitude can be compared with the dimensional reduction of the six-scalar $E_{7}$ supergravity amplitude. The latter can be found from a scalar component of $\mathcal{N}=4$ SYM Next-to-Maximally-Helicity-Violated (NMHV) amplitude, conveniently written as a sum of the so-called R-invariants, multiplied by an MHV prefactor [18,

$$
\mathcal{A}_{6}^{\mathrm{NMHV}}=\mathcal{A}_{6}^{\mathrm{MHV}} \times \sum_{3 \leq s+1<t \leq 5} R_{6 s t}
$$


and by making use of the KLT relations [2, 3]. It is then straightforward to check, again by choosing a range of numerical values for external momenta, that the resulting pure scalar amplitude in fact agrees with the squared BLG amplitude (12).

For higher-point amplitudes it would be possible to prove, along the lines of [19], that (12) holds if there were Britto-Cachazo-Feng-Witten (BCFW) recursion relations [20, 21] for the $E_{8}$-theory. Recursion relations for ABJM theories, and thus BLG theories, have been proven in 14]. The key step is proving that the superamplitude falls off sufficiently fast for large deformations of the momenta under a complex non-linear shift: $\hat{\mathcal{A}}\left(\left\{\lambda_{1}(z), \lambda_{l}(z)\right\}\right) \sim \mathcal{O}(1 / z)$ as $z \rightarrow \infty$ with $\lambda_{1}(z)=\frac{z+z^{-1}}{2} \lambda_{1}-\frac{z-z^{-1}}{2 i} \lambda_{l}, \lambda_{l}(z)=\frac{z-z^{-1}}{2 i} \lambda_{1}+\frac{z+z^{-1}}{2} \lambda_{l}$ and similar shifts for the Graßmann parameters. The proof of a sufficient fall-off for $E_{8}$ superamplitudes does not currently exist. However, it is possible to naively apply the method of [14] and use the fourpoint amplitude (11) to construct a candidate six-point superamplitude in $E_{8}$ supergravity. We find that the relevant scalar component, $M_{6}\left(\xi_{1} \bar{\xi}_{2} \xi_{3} \bar{\xi}_{4} \xi_{5} \bar{\xi}_{6}\right)$, of this superamplitude agrees with the amplitude calculated by squaring the numerators (12). This shows that at least to six points the BCFW recursion relations of [14] hold for the $E_{8}$-theory.

\section{Outlook}

In order to confirm the proposed "double-copy" relations for the $E_{8}$-theory, it would be very useful to prove in general the BCFW relations for the three-dimensional supergravity. Relatedly, numerator identities for YM and squaring relations for gravity have been conjectured to extend to all-loop diagrams [4], and it would be interesting to check whether similar relations hold for the three-dimensional Chern-Simons and gravity theories beyond tree-level. If this does indeed hold it would demonstrate the existence of a hidden three-algebra structure in three-dimensional gravity. This is interesting as a non-trivial model for similar structures in four dimensional gravity, particularly with regard to knotty issues of quantum gravity, and as an important intermediary step to two-dimensions where gravity is known to posses infinite dimensional symmetries [22, 23].

\section{Acknowledgements}

We thank N. Beisert, G. Bossard, F. Loebbert, R. Monteiro, H. Nicolai, D. O'Connell, and R. Roiban for helpful discussions. We would further like to thank the organizers of the Nordita program on Exact Results in Gauge-String Dualities, where parts of this work were presented, for their hospitality. Finally, TMcL would like to thank CPTh, Ecole Polytechnique for their hospitality during the completion of this work.

\section{References}

[1] Z. Bern, J. Carrasco and H. Johansson, "New Relations for Gauge-Theory Amplitudes", Phys.Rev. D78, 085011 (2008), 0805.3993.

[2] H. Kawai, D. Lewellen and S. Tye, "A Relation Between Tree Amplitudes of Closed and Open Strings", Nucl.Phys. B269, 1 (1986).

[3] F. A. Berends, W. Giele and H. Kuijf, "On relations between multi - gluon and multigraviton scattering”, Phys.Lett. B211, 91 (1988).

[4] Z. Bern, J. J. M. Carrasco and H. Johansson, "Perturbative Quantum Gravity as a Double Copy of Gauge Theory", Phys.Rev.Lett. 105, 061602 (2010), 1004.0476.

[5] J. Bagger and N. Lambert, "Modeling multiple M2's", Phys. Rev. D75, 045020 (2007), hep-th/0611108

[6] A. Gustavsson, "Algebraic structures on parallel M2-branes", 0709.1260. 
[7] J. Bagger and N. Lambert, "Gauge Symmetry and Supersymmetry of Multiple M2-Branes", Phys. Rev. D77, 065008 (2008), 0711.0955.

[8] M. Gunaydin and N. Warner, "Unitary supermulitplets of $O \operatorname{sp}(8 \mid 4, R)$ and the spectrum of the $S^{7}$ compactification of eleven-dimensional supergravity", Nucl.Phys. B272, 99 (1986).

[9] Y.-t. Huang and A. Lipstein, "Amplitudes of 3D and 6D Maximal Superconformal Theories in Supertwistor Space", 1004.4735

[10] O. Aharony, O. Bergman, D. L. Jafferis and J. Maldacena, "NN$=6$ superconformal Chern-Simons-matter theories, M2-branes and their gravity duals", JHEP 0810, 091 (2008), 0806.1218 .

[11] M. Van Raamsdonk, "Comments on the Bagger-Lambert theory and multiple M2-branes", JHEP 0805, 105 (2008), 0803.3803.

[12] T. Bargheer, F. Loebbert and C. Meneghelli, "Symmetries of Tree-level Scattering Amplitudes in N=6 Superconformal Chern-Simons Theory", Phys.Rev. D82, 045016 (2010), 1003.6120.

[13] A. Agarwal, N. Beisert and T. McLoughlin, "Scattering in Mass-Deformed N $\geq 4$ Chern-Simons Models", JHEP 0906, 045 (2009), 0812.3367.

[14] D. Gang, Y.-t. Huang, E. Koh, S. Lee and A. E. Lipstein, "Tree-level Recursion Relation and Dual Superconformal Symmetry of the ABJM Theory", JHEP 1103, 116 (2011), 1012.5032.

[15] N. Marcus and J. H. Schwarz, "Three-Dimensional Supergravity Theories", Nucl. Phys. B228, 145 (1983).

[16] P. Breitenlohner, D. Maison and G. W. Gibbons, "Four-Dimensional Black Holes from Kaluza-Klein Theories", Commun.Math.Phys. 120, 295 (1988).

[17] S. Mukhi and C. Papageorgakis, "M2 to D2", JHEP 0805, 085 (2008), 0803.3218.

[18] J. Drummond, J. Henn, G. Korchemsky and E. Sokatchev, "Dual superconformal symmetry of scattering amplitudes in N=4 super-Yang-Mills theory", Nucl.Phys. B828, 317 (2010), 0807.1095

[19] Z. Bern, T. Dennen, Y.-t. Huang and M. Kiermaier, "Gravity as the Square of Gauge Theory", Phys.Rev. D82, 065003 (2010), 1004.0693.

[20] R. Britto, F. Cachazo and B. Feng, "New Recursion Relations for Tree Amplitudes of Gluons", Nucl. Phys. B715, 499 (2005), hep-th/0412308.

[21] R. Britto, F. Cachazo, B. Feng and E. Witten, "Direct Proof Of Tree-Level Recursion Relation In Yang-Mills Theory", Phys. Rev. Lett. 94, 181602 (2005), hep-th/0501052.

[22] R. P. Geroch, "A Method for generating new solutions of Einstein's equation. 2", J.Math.Phys. 13, 394 (1972).

[23] B. Julia, "Group Disintegrations", Conf.Proc. C8006162, 331 (1980). 